\title{
Senior Citizen Assistive device
}

\author{
${ }^{1}$ Ms. Varsha Pathak, ${ }^{2}$ Mrs. Ekata Mehul \\ ${ }^{I}$ Department of Electrical engineering, $M S U$, \\ ${ }^{2}$ Head eiTRA EINFOCHIPS Training \&Research Academy
}

\begin{abstract}
-
The demographic change accompanied with fast changing family structure due to forces like urbanization and migration, necessitate requirement of support, care and treatment of older persons. With massive increase in older population and ageing people, unprecedented challenges are found in the field of health care, infrastructure support, quality of life, etc. Technology can help these people by such system which should provide security, interaction with relatives and it should be cost effective.

The objective of this project is to make one simple and cost effective system which will provide all facility needed by old man and they can overcome some common problems with old age like dependency, loneliness and forgetfulness. Controlling all appliances using remote will reduce dependency and interaction with relative will try to make more in contact with relatives. Special Locator Mode will help them to locate their own things put by themselves. There is special wireless network of sensor which helps to monitor some important health parameter which is important in this age. This project is little step in making of whole system which will be very helpful to the old age people.
\end{abstract}

\section{INTRODUCTION}

We live in a world where everything can be controlled and operated automatically, but there are still a few important sectors in our country where automation has not been adopted or not been put to a full-fledged use, perhaps because of nobody think in that direction seriously. One such field is that of "ELDERTRONICS". Previously discussed only in expert circles, "ELDERTRONICS" was firstexposed to a broader public in 2009, to be more exact, at the Consumer Electronics Show (CES) in Las Vegas, which many people consider the most important technology fair in the world. Some 10 million Indian citizens have arthritis and over 05 million are affected by hearing loss. To date, they have all been largely ignored by the entire electronics industry. We try to make little step in this direction which make their life easier. over this project to ease the life especially as far as old age people are concerned. Our basic aim to work over this project is to shape the existence of science, especially engineering into its genuine shape, i.e. to ease our lives. Thus we have determined to work over the project which is bolt from the blue.

This project aims at comfort as well as security of lives, including the memorandum of daily execution able works. Today our lives are totally occupied by nationally scheduled time tables.

Therefore there is an urge to proceed in this direction to ease the lives by creating something very subservient.

\section{PROPOSED MODEL OF THE SYSTEM}

The proposed system is embedded system which will try to solve all their problems at a certain extent. It will also reduce effort of caretakers. They can easily monitor status of loved one and take necessary steps to make it better. This whole system will take care of security and give protective environment which help old age to live more freely in their own homes without support of others. Cameras at door and remote operation of all home appliances including fan and light will reduce their work. Good operating system will also simplify to understand and operate whole system. Touch screen will help them to operate whole system by single touch. Large emergency button will help them in critical time. Online access of their status and report of critical parameter will help their doctor to update their medicine. Special Locator Mode will help them to find their thing which is kept and forgot. This device has interface with large screen which helps them to easily communicate with their loved ones. This system has both quality of portability and simplicity. So, this whole system will become perfect solution for old age people who are alone in their homes. 
IOSR Journal of Engineering

May. 2012, Vol. 2(5) pp: 1024-1028

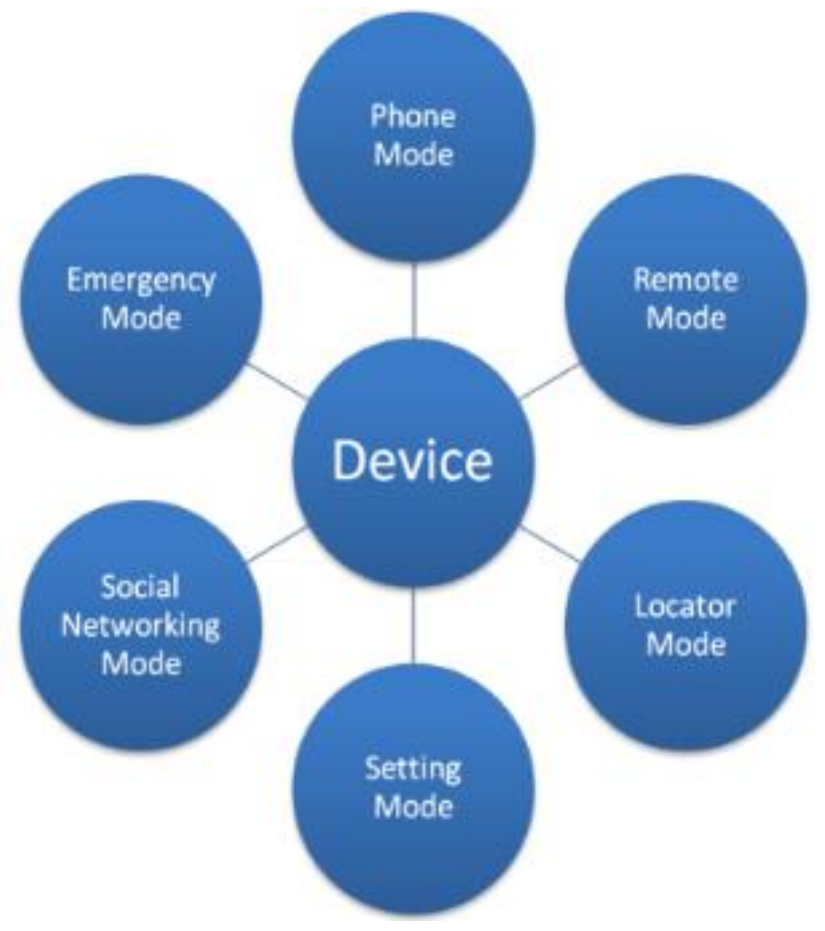

Fig.1 Various Modes of device

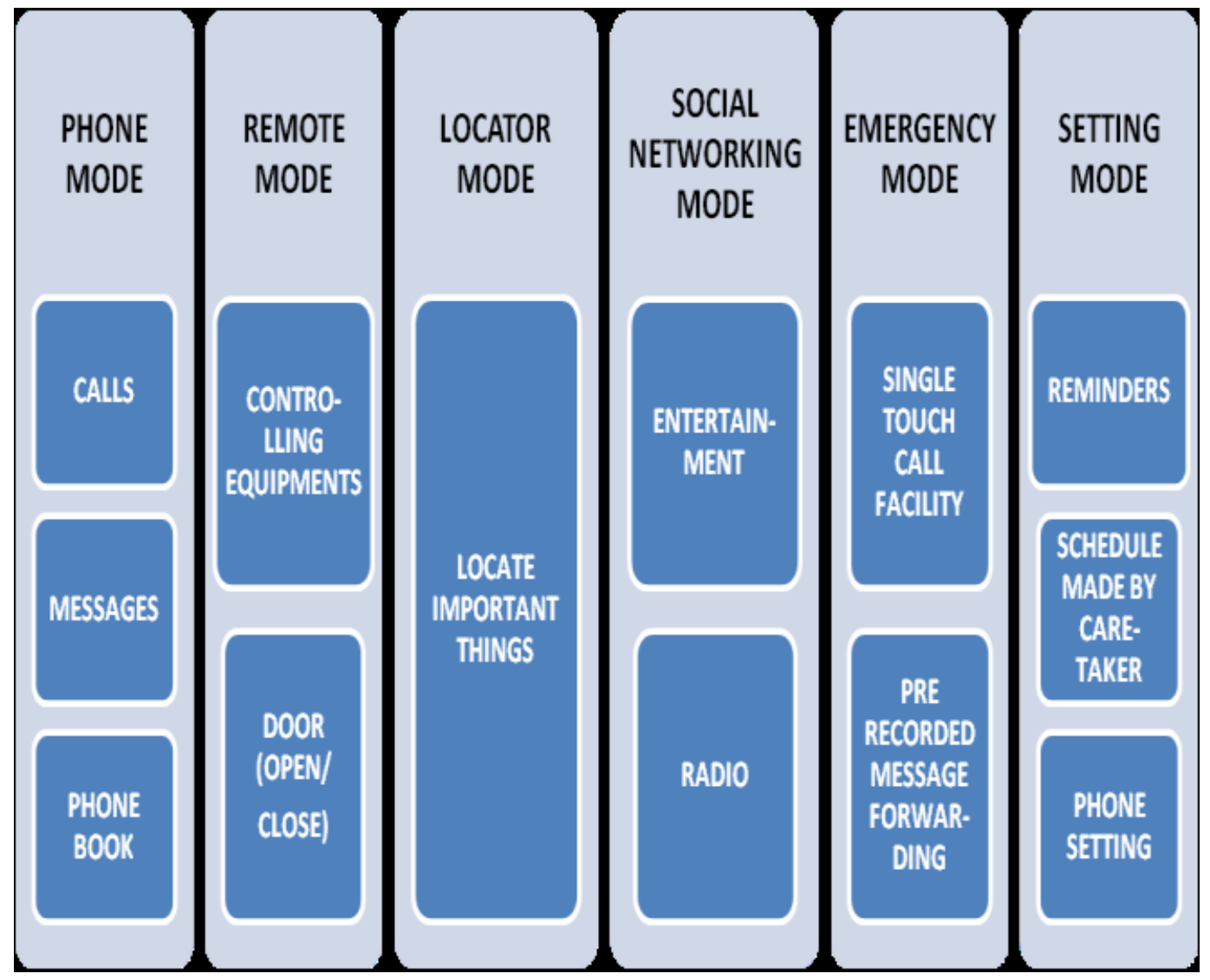

Fig. 2 Modes in detail 


\section{PHONE MODE}

It supports all features of mobile phone like calling or messaging someone. There is feature of voice tag which eliminates the need of searching the phonebook to call anyone. We can call by just speaking his/her name. Voice tag is very useful for them because old one faces problem to type the mobile number or finding their names. So they can call easily without any problem. Touch screen is provided for ease of use. No need to press button to operate phone and its functionalities. By this mode, they can stay connected with loved ones, relatives, their friends or when they need to contact any other person for some work so they can easily communicate with them. So this feature can avoid dependency. This feature is support by Portable Controlling Device. We have also facility that when call came his/her photo is shown by wall mounted device so they can easily recognize them

\section{REMOTE MODE}

Remote mode provides old one easy operating of appliances. It supports the controlling of domestic equipment like T.V, AC, Fan, Light etc.. This mode also support feature of open/close door which is very useful for them. If someone at door and press bell then his/her photo is taken by camera and displayed on wall mounted device. So, they can recognize them and open/close door whatever they want. This mode will help to reduce dependency on others because we can operate light/fan without any problem. Display will show position of light/fan and by just pressing on them we can on/off them. It will also indicate if main door is left open for more than predefined time which provides security.

\section{LOCATOR MODE}

Generally old ones have problem with memory power. So they often forget the places of their important things. So this mode is very helpful to them. This mode is basically used to find important things which is put and forgot by them. Here we use the device as transmitter and things are receivers. When the receiver catches the particular frequency transmitted by device then it will respond to it by producing the loud beeps. So they can find the things by finding the beep direction. So there is no need to move to find the things. So this mode is very helpful to old ones. This mode is supported by both Wall mounted device and portable device so if they forgot where is the portable device they can find it by use of wall mounted device. Things are shown in screen and by just touching them alarm is activated and again touch will off it.

\section{SOCIAL NETWORKING MODE}

When people entered into old age, their family at work makes them fill lonely. Involvement of their family and friends will help them to live happily which helps them to live longer. This mode will help them to easily contact with family and friends. They can do video calling and chatting with them. Their loved one can send mail, pictures, and videos to them. It will be very easy for them to operate mail and internet. 24 hour NEWS will be available to them so they will in touch with outer world. There is also facility to store favorite songs and listen when they want to. There are some games which help them to remain mentally active. So, this mode provides connection with outer world and entertainment for them which helps to remove loneliness.

\section{EMERGENCY MODE}

No one knows what will happen in next moment. So there is need of emergency for old ones. There is one push button on the backside of the device by pressing that button will send prerecorded voice message to five of their relative set by caretaker. This will help in emergency and make possible to get treatment when most needed.

\section{SETTING MODE}

This mode is very important which sets all setting of other modes. This mode is possible to access online and password protected so their caretakers can easily access them anywhere and monitor position of their loved ones. This mode is supported by only wall mounted device. In this mode there are different setting options. We can set reminders of different things like take medicines or family birthdays or doctor's appointment. Which helps them to regularly take medicine and keep record that they take medicine or not with help of 'Pill Box'? There is also record of different parameters which are monitor by different sensors like blood pressure, glucose level and weight of them. This database will be seen online and take printout out of it. There are other options of setting of different modes like phone modes, remote mode, locator mode etc. We can set ringer and vibration and set which things to control with remote. Caretakers will monitor this mode and check wellness report of their loved one. 


\section{BASIC MODEL OF THE SYSTEM}

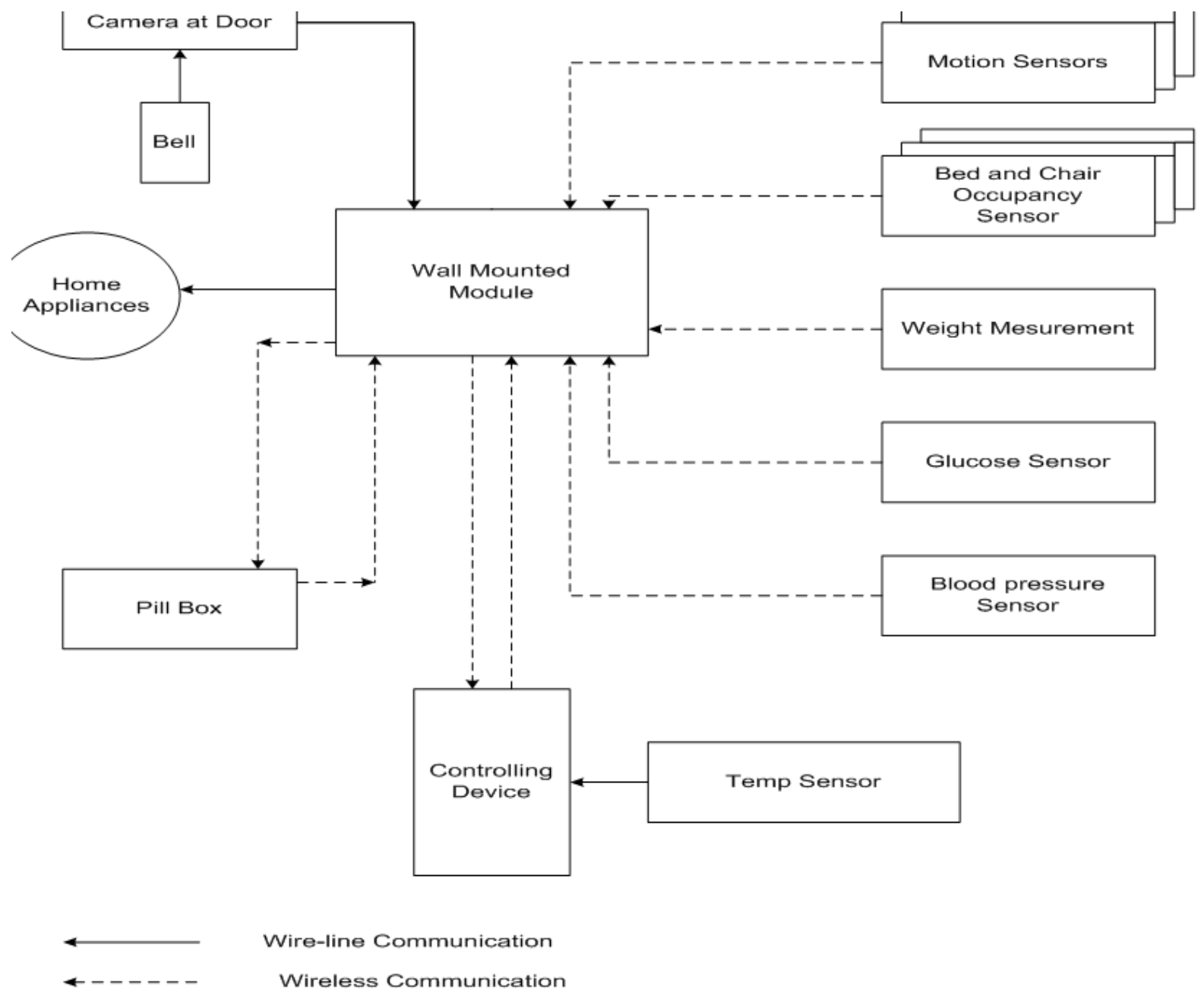

Fig.3 System Model Wall Mounted Module

This is main module of the system. Whole system is controlled by this device. We can give command using controlling device but operation of all devices is control by wall mounted device. This module is either in form of existing T.V. or Computer Monitor. This is used to view different options of system as well the images taken by the camera at outside the door to inform about person at door. This is beneficial for visually impaired elders. This module also gives $24 \times 7$ news, Entertainment and views the images sent by caregiver via internet.

\section{Controlling Device}

This module is small Touch screen which can be easily mounted on hands like wrist watch so than one can move easily with it. This device can communicate with wall mounted device wirelessly to operate the options viewed by wall mounted device. This device also views the same options so that one can easily operate it with touch without seeing on T.V. In this device we have a GSM module with voice tag facility so it can be used to call their loved one. Using this device we can make our home appliances remote controlled and locate the daily used things of old ones. Sensors There are different types of sensors which are used to monitor the health reports like body temperature, blood pressure, glucose, extreme sitting on chair, medicine reminder etc. and send the health report periodically to the care givers via internet and also stores the complete report in wall mounted device. These sensors are used to monitor complete health of old age and take necessary steps.

\section{Camera}

This camera has in built JPEG engine. This camera takes the images when motion is detected outside the door using motion sensor and convert it into JPEG format and sends it to wall mounted device to inform old one about the person present outside the door. This Camera will add security feature in this system. 


\section{Home Appliances}

These are the routine appliances which they frequently used like light, fan, air conditioner etc. Which are to be operated remotely using controlling device. This feature will help old one to operate door as well so they can control whole house using controlling device only.

\section{ADVANTAGES}

1. Easy-to-use, patient centric design.

2. Simple, intuitive Touch Screen.

3. Large, high resolution Graphics LCD.

4. Special Locator Mode which overcomes the shortcomings of forgetfulness in old age.

5. Device design is Modular so that it is possible to provide the features as per the demand.

6. Social Networking mode for talkative persons.

7. Remote mode makes life easier for physical impaired person due to old age.

8. Device communication is wireless with 30 meter range so all operation can be done from anywhere in house.

9. No technical expertise required.

10. Improve memory and cognition with interactive games and trivia.

11. Keep track of your own weight, blood sugar and blood pressure.

12. Emergency help button.

13. Family and caregivers can view and monitor the data online.

\section{ACKNOWLEDGEMENT}

The authors would like to acknowledge Ekata Mehul of eInfochips for her contribution to this work and for reviewing the paper

\section{REFERENCES}

[1] Muhammad Ali Mazidi, Janice Gillispie Mazidi, Rolin D. Mc Kinlay,

[2] The 8051 Microcontroller \& Embedded Systems, Pearson Education Inc. 2nd Edition, 2008.

[3] Kenneth J Ayala, the 8051 Microcontroller Architecture, Programming \& Applications, Penram International, 2nd Edition, 1996.

[4] Ramakant Gayakwad, Operational Amplifiers Linear Integrated Circuits, Prentice Hall of India, 3rd Edition.

[5] Hands-on Zigbee, By Fred Eady 